\title{
Springback Simulation in Air Bending of Titanium Alloy Sheet Using Solid Shell Element
}

\author{
FU Xue-song ${ }^{a}$, LI Kang ${ }^{b}$, SUN Weic, LIU Cheng-fang ${ }^{d}$, CHEN Guo-qing ${ }^{e}$ *, \\ ZHOU Wen-long ${ }^{\dagger}$
}

Key Laboratory of Solidification Control and Digital Preparation Technology (Liaoning Province), School of Materials Science and Engineering, Dalian University of Technology, P.R. China

axsfu@dltu.edu.cn, bliking@mail.dlut.edu.cn, csunwei@dlut.edu.cn, d1010456208@qq.com, egqchen@dlut.edu.cn, 'wlzhou@126.com

Keywords: springback; air bending; solid shell element; mesh discretization; integration scheme. Abstract. Accuracy improvement of springback prediction is a major concern in finite element simulation of sheet metal bending process, especially for the materials with high elastic recovery. This work adopts solid shell finite element for springback simulation and improves the simulation accuracy by means of rationalizing mesh discretization and through-thickness integration scheme. Meanwhile, air bending experiment of titanium alloy Ti-6Al-4V was carried out to examine the simulation result. Research results demonstrate that solid shell element has better performance than thick shell element in springback simulation. The accuracy can be improved evidently by extending the mesh refinement area to 2 times of sheet thickness from the bending corner. The optimal number of integration points varies with the bending radius and the limited error tolerance.

\section{Introduction}

Prediction of following springback is crucial in mold design to obtain parts with higher dimensional accuracy, especially for high-strength materials. Traditional empirical adjustment for springback is expensive and time-consuming, and not applicable to products of complex geometries. Nowadays, the finite element analysis of springback has been proven as a well-established tool for design engineers due to its lower cost and shorter delivery periods. For the purpose of reducing error in bending process, it is necessary to make efforts on improving the accurateness of springback simulation, especially for the materials with high strength $(\sigma s)$ and low Young's modulus (E) because of their high elastic recovery.

A crucial point in the computational modeling of elastoplastic behavior is the proper choice of the finite element formulation[1]. Several elements with unique properties have been tried to simulate the sheet metal bending process and springback behavior. Among the elements, four-node shell element[2-7] and eight-node solid element[8-12] are the most popular because of their respective outstanding advantages. In the last decade, reduced enhanced solid shell (RESS) element developed by Alves de Sousa [13-15], which has both advantages of shell element and solid element, has aroused a lot of interest. The application of solid shell elements has practical significance on the accurate simulation of sheet metal bending process. For the attractive superiority of solid shell element in 3-D and double-sided contact simulations, many researchers have made efforts on its property enhancement. Parente[16] tested solid shell element with enhanced assumed strain (EAS) method in simulating the forming of an S-Rail industrial component, showing that the enhanced element can avoid the locking effect and provide reliable solution. Schwarze[17] used solid shell element with EAS and assumed natural strain (ANS) method to analyze the parameters of deep drawing and predict the springback.

Except the element formulation, meshing method and the number of integration points through the thickness direction (NIP) also have a significant influence on the simulation solutions and computation time. Optimal mesh density, especially in the bending zone, is a balance between accuracy of simulation solutions and computing efficiency. S.W. Lee[18] indicated that the optimal element sizes are 0.2 and 0.15 of the die corner radius for the blank and the tool corners respectively. 
W.L. $\mathrm{Xu}[19]$ concluded that springback simulation is very sensitive to the number of elements in contact with the die radius and 5 7 elements on the radius of the tooling is optimal. T. Meinders[20] suggested an angle of $9^{\circ}$ per element in the corner is necessary to minimize the discretization error in springback analysis. In this work, optimal mesh method in bending corner zone will be discussed in terms of sheet thickness.

Integration rules require a significant number of integration points (NIP) in element thickness to reflect the nonlinear stress and strain when a material undergoes elasticplastic deformation. NIP required for accurate springback simulation using shell elements is always controversial. $\mathrm{Li}$ and Wagoner[21, 22] recommended use of 25 integration points (IP), with up to 51 IP needed to ensure accuracies of $1 \%$, in general springback simulation. However, several subsequent literatures report less IP is optimal, including 9 IP [23] and 7 IP[19]. Afterwards, an intensive study was done by Wagoner and $\mathrm{Li}[24]$, they pointed out that NIP varies with the process parameters, integration rules and material properties in springback simulation of a beam bent under tension. At present, seldom papers report the rules of integration schemes for springback simulation when solid shell element is used.

In this paper, springback simulation of Ti-6Al-4V titanium alloy sheet with high ratio of $\sigma \mathrm{s} / \mathrm{E}$ is studied using solid shell element. The research emphasis is the influence of meshing method and the optimal NIP choice on the accuracy improvement of springback prediction. Furthermore, experimental measurement of springback angle is carried out to examine the rationalization of simulation scheme.

\section{Experimental procedure}

Experimental molds consist of " $U$ " type-bending punch and die with $60^{\circ}$ as bending angle, as shown in Fig.1 and Fig.2. There are three sets of molds with different bending radius $(R)$ of $7.5 \mathrm{~mm}$, $15 \mathrm{~mm}$ and $22.5 \mathrm{~mm}$, respectively. Except the $R$, all other influential parameters remain the same to exclude their effects on the springback analysis. Molds are fixed on a press machine which has the ability of speed adjustment and feedback.

The dimension of the bending specimens is $140 \mathrm{~mm} \times 30 \mathrm{~mm} \times 1.5 \mathrm{~mm}$. The mechanical behavior of titanium alloy is identified by tensile test. The stress-strain curve is transformed into true values and then fitted as Swift's isotropic strain hardening law:

$$
\sigma=K\left(\varepsilon_{0}+\varepsilon_{P}\right)^{n}
$$

where $\sigma$ is the flow stress; $K=1797 \mathrm{MPa}$, the hardening coefficient; $\varepsilon_{0}=0.04168$, the offset strain; $\varepsilon_{P}$ is the plastic strain; $n=0.1929$, the hardening exponent. The Young's modulus is $120 \mathrm{GPa}$. The poission ratio $v$ is 0.3 .

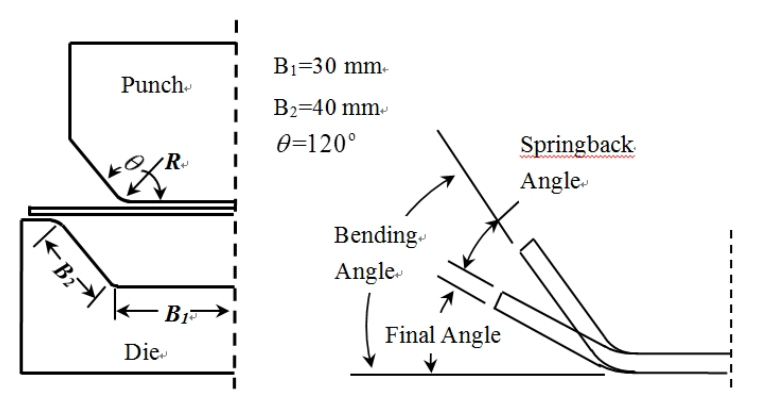

Fig. 1 Schematic of experimental molds and springbacks 


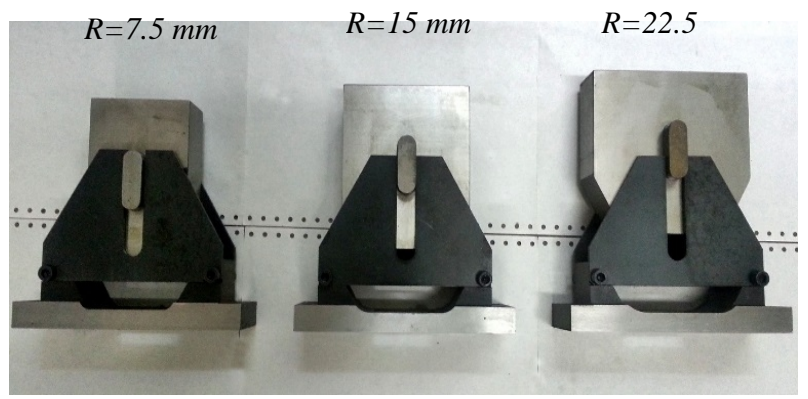

Fig. 2 Experimental molds with different bending radius

\section{Finite element modeling}

\section{Solid shell element}

For simulation of sheet metal bending, especially small $R / t$ ratios $(<5)$ or large stretching, accurate solution can't be provided by using shell elements even if very fine mesh ${ }^{[25]}$. Thus, element formulation using full 3D constitutive laws is a reasonable choice. Solid element meets the 3D constitutive laws but it will result in computationally expensive due to the necessarity of multiple layers. Therefore, solid shell elements possessing both advantages of shell elements and solid elements become very popular in shell-like structure simulation and appropriate for dealing with double-sided contact problem. Solid shell element bases on eight-node solid elements' topologies with user-defined $N_{\text {IP }}$ through the element thickness (Fig.3). Differing from shell elements, solid shell elements have only translational degrees of freedom and not based on plane stress assumptions.

Sheet metal bending simulation usually associates with plastic strain. Therefore, element locking problems must be solved to assure the solution reliability. In this paper, solid shell element with selective reduced integrated (SRI), enhanced assumed strain (EAS) and assumed natural strain (ANS) method, called as M-RESS element developed by Alves de Sousa[15, 26], is used. The SRI method can avoid volumetric locking; the ANS method is used to overcome shear locking; the EAS method is considered to improve the bending performance and reduce volumetric locking. Furthermore, only using a single enhancing variable in EAS offers a good combination between accuracy and computational efficiency.

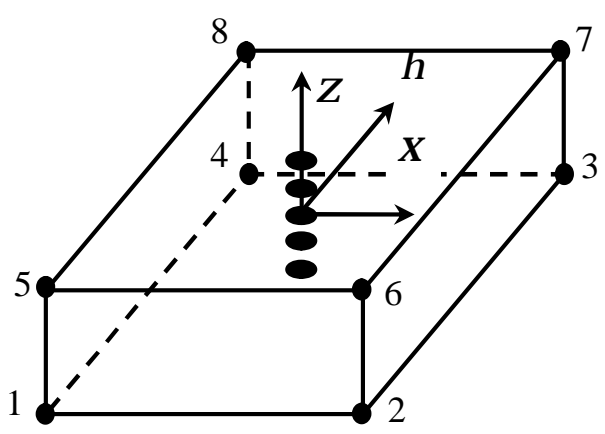

Fig. 3 Solid shell element with the user defined $N_{I P}$

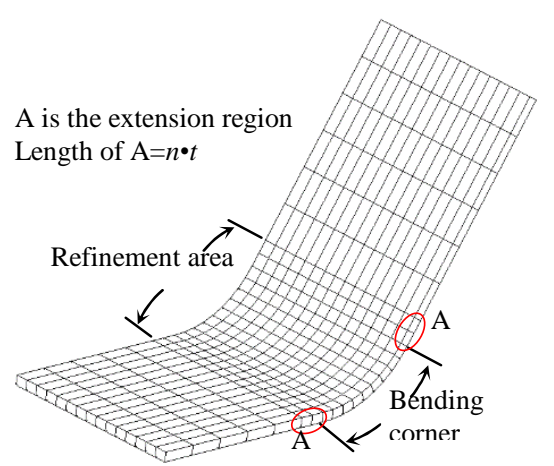

Fig. 4 Schematic of meshing methods

\section{Meshing method and NIP}

The dies are modelled as analytic rigid surfaces and the sheets are assumed to be free of residual stress before bending. Since springback following air bending involves no contact and mild nonlinearities, Marc/mentat implicit FE-package is used. 
Meshing method of the sheet has a great influence on the calculation of stress state in bending. During bending deformation, high stress gradients take place in the region around the tool radius, so this region should be modeled by a fine mesh. To determine the optimum size of refinement region, the sheet is discretized using fine meshes in bending corner with an extended length of $n(n=0 \sim 3)$ times of sheet thickness $(t)$ and coarse meshes in rest regions, as shown in Fig.4. The element length of the fine meshes and coarse meshes are $0.12 R$ and $0.4 R$, respectively. Meanwhile, thick shell element is also employed to compare with M-RESS element. Both elements are set 49 as the $N_{\text {IP. }}$.

Based on the determination of the rational refinement region, study on through-thickness integration schemes is launched. The numerical integration is done using Simpson's rule. $N_{\text {IP }}$ must be odd, the set value for $N_{\mathrm{IP}}$ varies from 3 to 49 in springback simulations.

\section{Results and discussion}

Fig. 5(a) shows the experimental result of Ti-6Al-4V sheets after unloading. The springback angles of experimental measurements are $5.3^{\circ}, 15.7^{\circ}$, and $23.4^{\circ}$ for bending radius of $7.5 \mathrm{~mm}, 15.0 \mathrm{~mm}, 22.5$ $\mathrm{mm}$, respectively. It can be seen that springback angle increases with bending radius. This tendency also appears in the simulation results, as shown in Fig. 5(b). As observed, the simulation results using M-RESS elements are generally closer to the experimental measurements than thick shell elements for all bending radius. The reason is the differences on the element formulations and bending performance. M-RESS element uses EAS formulation for normal thickness strain. However, thick shell element hasn't the ability to present the stress state in thickness direction.

Furthermore, the accuracy of springback prediction has a close relation with mesh refinement in the vicinity of bending corner. Fig. 6 shows the effect of the extended length on springback error between experimental and simulation results, the extended length from bending corner is $n$ times of sheet thickness. The springback error is given by

$$
\operatorname{Error}(\%)=100 \times\left|\frac{\theta-\theta \exp }{\theta \exp }\right|
$$

where $\theta$ and $\theta_{\text {exp }}$ are the springback angles of simulation results and experimental measurements, respectively.
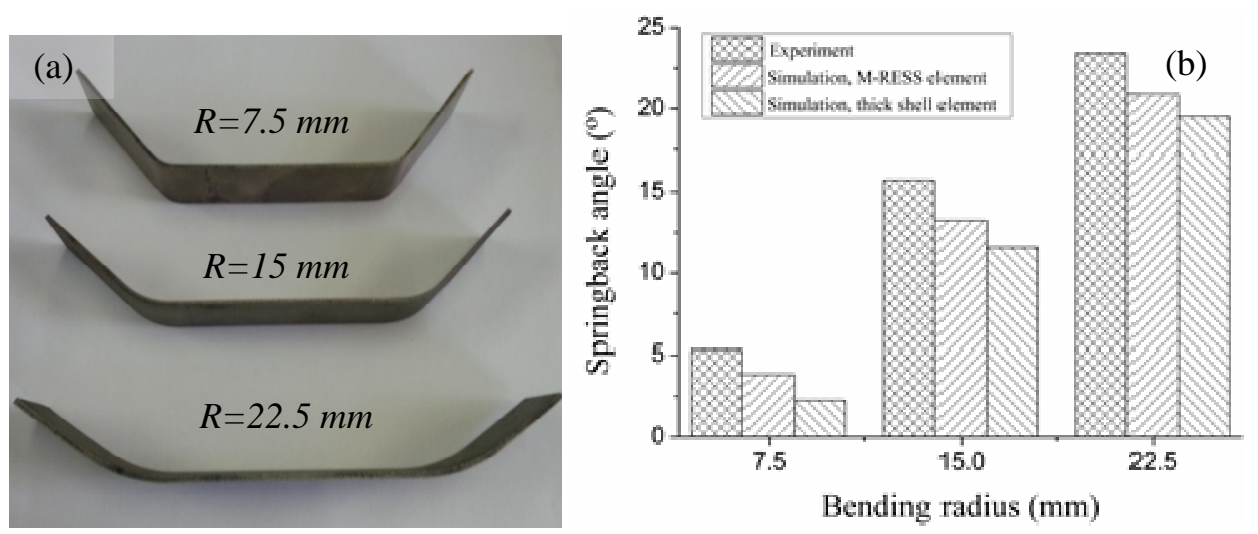

Fig. 5 Ti-6Al-4V titanium alloy sheets after unloading (a) and springback angles of experimental and simulation

results for different bending radius (b)

It demonstrates that the error decreases evidently with the increase of $n$ for both M-RESS element and thick shell element. However, the accuracy improvement will not be evident when $n$ is above 2 . Therefore, the optimum value of $n$ should be set as 2 as computing cost is taken into consideration. Now, it can be concluded that the optimum meshing method is refining the mesh in the bending corner with an extended length of $2 t$.

The phenomenon that the meshing method affects the simulation accuracy is attempted to be 
explained by discussing the stress-strain distribution which has a direct influence on springback. Fine mesh of EAS solid elements is implemented to obtain the accurate stress-strain field. Fig. 7 and Fig. 8 show the distributions of equivalent mises stress and equivalent plastic strain as the Ti-6Al-4V sheets are completely compressed. The figures show that high stress gradients and plastic deformation not only appear in the bending corner but also in its vicinity regions. And the size of the vicinity, where high stress gradients take place, is about $2 t$ length from the bending corner. Therefore, it is necessary to use fine meshes in the bending corner with an extended length of $2 t$.
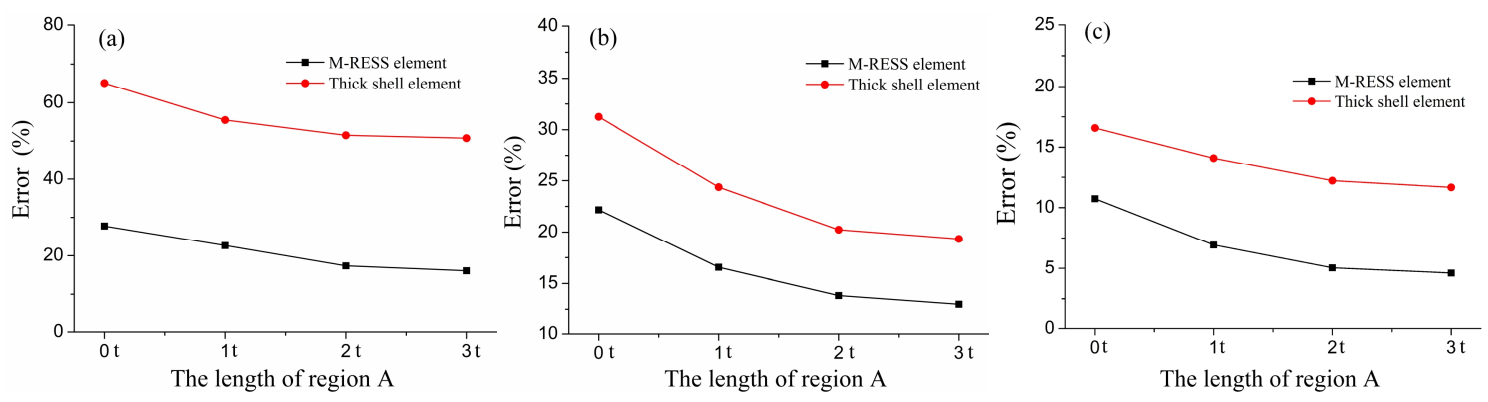

Fig. 6 Effect of mesh refinement region A (see Fig. 4) on the error of springback angles for different bending radius:

(a) $R=7.5 \mathrm{~mm}$; (b) $R=15 \mathrm{~mm}$; (c) $R=22.5 \mathrm{~mm}$. $t$ represents the sheet thickness
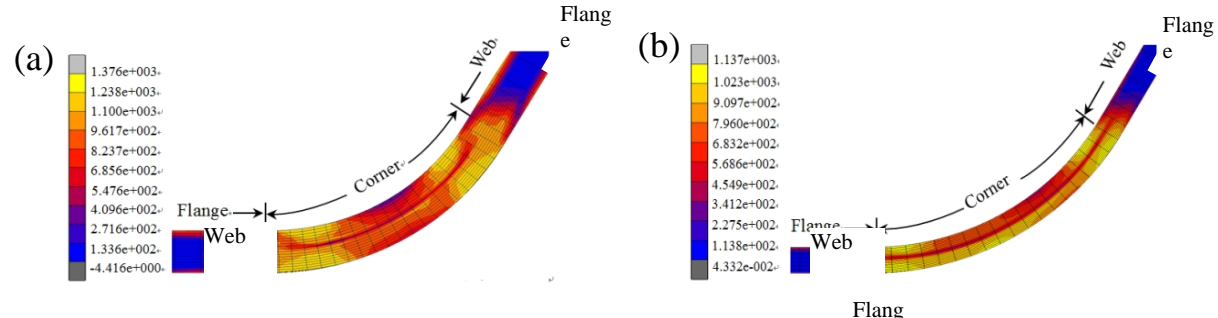

(c)

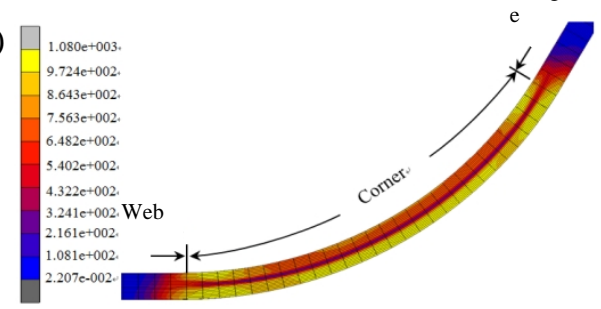

Fig. 7 Equivalent mises stress in bending area and its vicinity: (a) $R=7.5 \mathrm{~mm}$; (b) $R=15 \mathrm{~mm}$; (c) $R=22.5 \mathrm{~mm}$
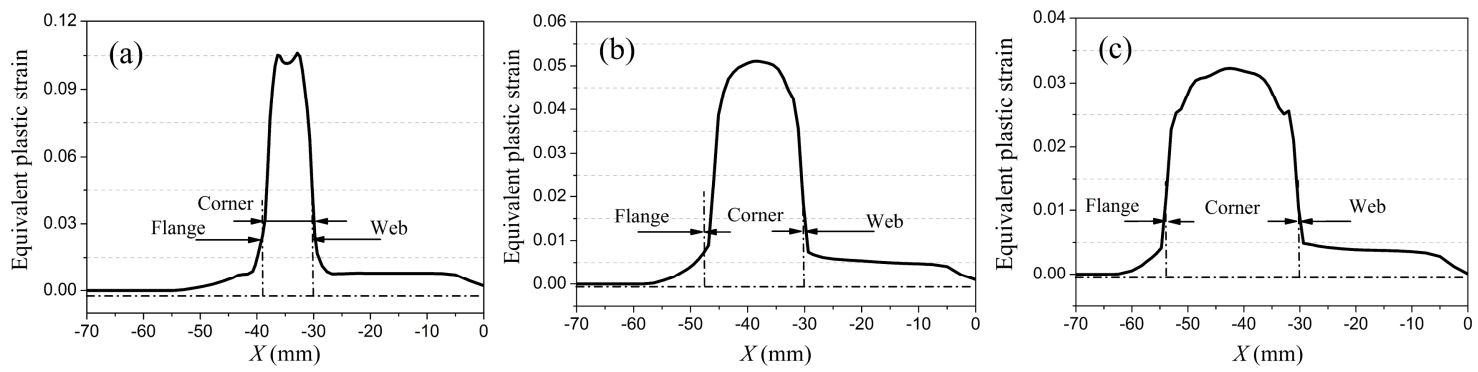

Fig. 8 Plastic strain versus $x$ coordinate for half a profile with different bending radius:(a) $7.5 \mathrm{~mm}$; (b) $15 \mathrm{~mm}$; (c) $22.5 \mathrm{~mm}$ 
Table 1 Required $N_{\text {IP }}$ for $5 \%$ and $1 \%$ error tolerance with different bending radius

\begin{tabular}{llll}
\hline $\begin{array}{c}R(\mathrm{~m} \\
\mathrm{m})\end{array}$ & 7.5 & 15 & 22.5 \\
\hline $5 \%$ & 11 & 9 & 7 \\
$1 \%$ & 17 & 13 & 11 \\
\hline
\end{tabular}

$N_{\text {IP }}$ is a significant parameter for springback simulation using solid shell element. Simulation results in Fig. 9 show the effect of $N_{\text {IP }}$ on the error for different bending radius. The error has a large fluctuation when $N_{\text {IP }}$ is set as small value. However, when a specific big $N_{\text {IP }}$ is adopted, the error converges to a stable value. Considering the computing efficiency, the optimal $N_{\mathrm{IP}}$ is the number when the error begins to stabilize in an allowed tolerance. For $5 \%$ and $1 \%$ tolerance, required $N_{\text {IP }}$ is showed in table 1. It shows that more $N_{\mathrm{IP}}$ is needed for smaller bending radius and limited tolerance.

The distributions of equivalent plastic strain through the thickness at the middle of bending zone are shown in Fig.10. As observed, the equivalent plastic strain increases with decrease of bending radius. The neutral layer (equivalent plastic strain equal to zero) has an inside displacement deviating from the middle surface. In sheet thickness direction, it appear highly nonlinear strain state. For accurately coping with the nonlinearity, more $N_{\mathrm{IP}}$ is required when bending radius is smaller. It can be speculated that the $N_{\text {IP }}$ required for $R / t<5$ is equal or greater than 11 and 17 for $5 \%$ and $1 \%$ tolerance, respectively.

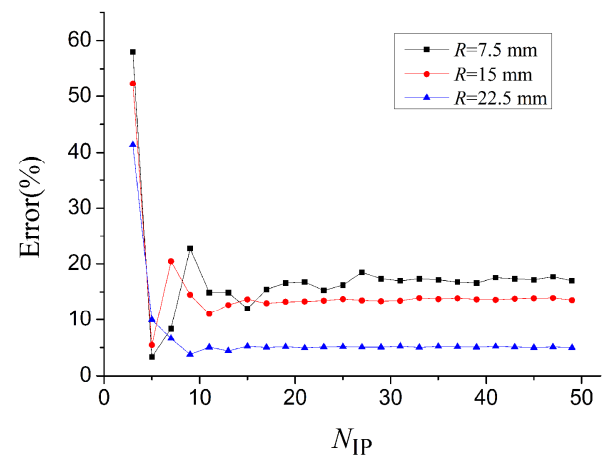

Fig. 9 Variation of springback error with $N_{\mathrm{IP}}$ for different bending radius

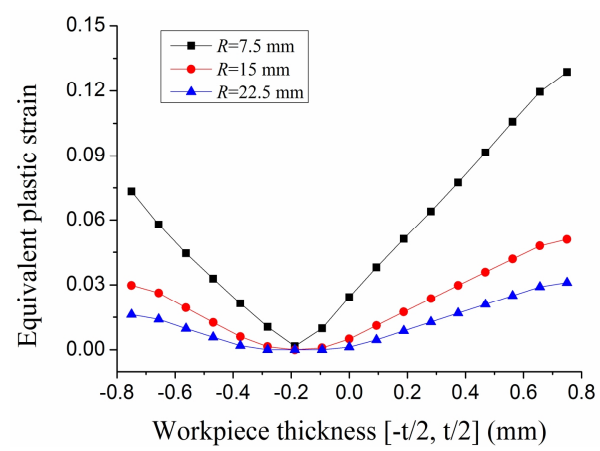

Fig. 10 Equivalent plastic strain distributions through the thickness at the middle of bending area 


\section{Conclusion}

Numerical simulation of springback following air bending of titanium alloy sheets has been performed. The following conclusions can be drawn:

(1) The M-RESS element has better performance than thick shell element in springback simulation.

(2) In order to improve the accuracy of springback prediction, it is optimal to extend the mesh refinement region to 2 times of sheet thickness from the bending corner.

(3) The optimal $N_{\text {IP }}$ varies with the parameters of bending process and the limited error tolerance. More $N_{\mathrm{IP}}$ is required for smaller bending radius and higher confidence.

\section{Acknowledgement}

This work was financially supported by the National Natural Science Foundation of China (No. 51175059), and also supported by China Postdoctoral Science Foundation (No. 2014M551074) and the Fundamental Research Funds for the Central Universities(DUT14RC(3)038).

\section{Reference}

1. S. Ghosh, J.M. Castro, J.K. Lee: Materials Processing and Design: Modeling, Simulation and Applications (Springer Verlag, 2004).

2. M. Lindgren: J. Mater. Process Tech. Vol. 186 (2007), p. 77-81.

3. M. Brunet, S. Mguil, and P. Pol: J. Mater. Process Tech. Vol. 80-81 (1998), p. 213.

4. P. Hartley, M.S. Tehrani, H.M. Naeini, H. Khademizadeh: Thin Wall. Struct. Vol. 44 (2006), p. 184.

5. G. Zeng, S.H. Li, Z.Q. Yu, X.M. Lai: Mater. Design, Vol. 30 (2009), p. 1930.

6. C. Gomes, O. Onipede, and M. Lovell: J. Mater. Process Tech. Vol. 159 (2005), p. 91-98.

7. N. Narasimhan, and M. Lovell: Finite Elem. Anal. Des. Vol. 33 (1999), p. 29.

8. Q.V. Bui, L. Papeleux, and J.P. Ponthot: J. Mater. Process Tech., Vol. 153-154 (2004), p. 314.

9. J. Paralikas, K. Salonitis, and G. Chryssolouris: Int. J. Adv. Manuf. Tech. Vol. 44(2009), p. 223.

10. J. Paralikas, K. Salonitis, and G. Chryssolouris: Int. J. Adv. Manuf. Tech. Vol. 47(2010), p. 1041.

11. J.H. Wiebenga, M. Weiss, et al : J. Mater. Process Tech. Vol. 213(2013), p. 978.

12. F. Heislitz, H. Livatyali, et al: J. Mater. Process Tech. Vol. 59 (1996), p. 59.

13. R.J.A. de Sousa, R.P.R. Cardoso, et al: Int. J. Numer. Meth. Eng. Vol. 62(2005), p. 952.

14. R.J.A. de Sousa, R.P.R. Cardoso, et al: Int. J. Numer. Meth. Eng. Vol. 67(2006), p. 160.

15. R.J.A. de Sousa, J.W. Yoon, et al: Inter. J. Plasticity Vol. 23 (2007), p. 490.

16. M.P.L.Parente, R.A.F. Valente, et al: Finite Elem. Anal. Des. Vol. 42(2006), p. 1137.

17. M. Schwarze, I.N. Vladimirov, and S. Reese, Comput. Method. Appl. M. Vol. 200(2011), p. 454.

18. S.W. Lee and D.Y. Yang, J. Mater. Process Tech. Vol. 80-81(1998), p. 60.

19. W.L. Xu, C.H. Ma, C.H. Li, W.J. Feng, J. Mater. Process Tech. Vol. 151 (2004), p. 217.

20. T. Meinders, I.A. Burchitz, et al: Int. J. Mach. Tool. Manu. Vol. 48 (2008), p. 499.

21. J.A. Meech, M.M. Veiga, M.H. Smith: Proceedings of the Second International Conference on Intelligent Processing and Manufacturing of Materials(IEEE Press, Honolulu, 1999).

22. K.P. Li, W.P. Carden, and R.H. Wagoner: Int. J. Mech. Sci., Vol. 44 (2002), p. 103.

23. V.T. Nguyen, Z. Chen, P.F. Thomson: P. I. Mech. Eng. C.-J. Mec. Vol. 218 (2004), p. 651.

24. R.H. Wagoner, and M. Li: Inter. J. Plasticity Vol. 23 (2007), p. 345.

25. R. Hauptmann, S. Doll, M. Harnau, K. Schweizerhof: Comput. Struct. Vol. 79 (2001), p. 1671.

26. R.P.R. Cardoso: Int. J. Numer. Meth. Eng. Vol. 75 (2008), p. 156. 